\title{
Electronic Health Records (EHR) of the Emergency Service of the Hospital de Clinicas: Case Study
}

\author{
Alvaro León Silvano, María E. García-Díaz, Diego P. Pinto-Roa, \\ Universidad Nacional de Asunción, \\ Asunción, Paraguay \\ \{aleon,mgarcia,dpinto\}@pol.una.py
}

and

\author{
David Ocampos N., Marcelo Pederzani \\ Universidad Nacional de Asunción, \\ Asunción, Paraguay \\ docampos@pol.una.py,mpederzani@med.una.py
}

\begin{abstract}
The Emergency Service management automation of the Hospital de Clínicas of the Universidad Nacional de Asunción is a problem addressed in this work since the patient's care in critical conditions must be accurate, appropriate, and efficient. The development of a management automation tool will generate benefits for both the patient and the hospital's target staff. With the automatization of the processes, it will be possible to achieve: (a) more time to devote to better patient care; (b) doctors will be able to spend part of their time analyzing the statistics and information, which can be generated through the application to perform investigations in the area of emergency care; (c) as well as to optimize the resources of both the staff and logistics used in the hospital. In this work, we present the development of an Electronic Health Records System management based on international health standards to achieve the proposed objective and good practices in the care processes in an area as sensitive as the Emergency Service. After more than 100 years of hospital history, for the first time, it is intended to automate the emergency service processes and generate online information quickly and efficiently. This system will help optimize patient care in the emergency area.
\end{abstract}

Keywords: Emergency Service, Application for Society, e-Health, Triage, RAC, Hospital Management, Electronic Health Records (EHR), Usability.

\section{Introduction}

Emergency services most often have an asymmetry between high demand for patient care and scarce resources available in these areas. This situation creates the effect that patients who come to the health center are not cared for timely. During the waiting time, the patient's condition may worsen. Under different conditions, they would have been favored for immediate care. This situation creates a sense of frustration and distress in the patient and their families as the time between the patient's arrival, and the care treatment is prolonged.

The Department of Emergency Services (DES) of the Hospital de Clinicas of the Universidad Nacional de Asunción (UNA) is one of the most sensitive and congested patients' health services. DES serves the most unprotected citizens and who do have no health insurance and without access to primary care. In this context, congestion in emergency services causes several problems for patients and health workers, such as ever-longer waiting times, increasing ambulance deviations, ever-prolonged stays, increased number of medical errors, hospital-acquired infections, re-admission, and increased patient mortality. These problems impact significant losses in economic resources that are further aggravated when all information collected and processed is done manually without the support of Information and Communication Technologies (ICTs) 1].

Currently, DES demands a great deal of time from health workers to make the efforts mentioned above and consume significant institutions' resources. A patient's admission into DES requires settling personal 
data and their health condition at that time. This scenario generates large quantities of information sheets written manually. In the same sense, searching for patient information on paper files also requires valuable time from DES staff. All these details make patients' waiting times very long, creating inconveniences typical of the care system and in breach of the international standard. Moreover, DES treats approximately 240 patients per day, resulting in average care of approximately ten patients per hour in the 24-hour service. Also, seven forms are commonly used per patient, and in one day, about 1680 units $[2]$.

Based on the abovementioned critical background, the main contribution of this work is to innovate and improve the service provided in DES using a computer system. The activities will be automated through a comprehensive management system, notably the Electronic Health Record (EHR) management, considering the widely accepted international standards in e-health. The designed and implemented system in this work is called SIGHU (Sistema Integral de Gestión Hospitalaria para Urgencias by its acronym in Spanish). This system implements the DES functions, where data are structured in the EHR for further processing and reviewing service statistics. These features based on the Paraguayan Ministry of Public Health 3 . help improve processes reducing waiting time in the care system. Note that these procedural manuals are based on the Spanish model of emergency processes 4 .

The organization of this work is as follows. Section 2 describes the theoretical framework of the work and some related works. In contrast, Section 3 presents the proposal for a solution to the problem raised. Section 4 describes the design and deployment of the system. Section 5 details the processes of usability and quality analysis. Section 6 describes the results and discussion. Section 7 mentions the Conclusions and future work.

\section{Theoretical Background}

The hospital management problem is still a global issue, and the emergency area is no stranger to this situation [5. Hospital management is susceptible to rules that are applied or not by the lack of financial resources. This issue limits the application of standards, from the computerization of physical and management records to the processes that infrequent be applied. In addition, it affects the lack of hospital resources such as instruments, medicines, reagents, among others 5]. A critical aspect is the management of health personnel. In many cases, they resist using electronic media (software and hardware), claiming various reasons such as lack of time and training in the use of technologies and mainly being in a department where the "urgency" is evident in most of the cases addressed. All these aspects pose a challenge that is addressed in eHealth [4]. This research discusses the case of UNA's School Hospital, which assists patients with lower resources in the country and reaches about 25000 patients per year.

The first formal definition of eHealth was made in 2001 by Gunther Eysenbach [6]. This definition proposes that eHealth is an emerging field at the intersection of medical computing, public health, and business. eHealth combines health services and information transmitted or improved over the Internet and related technologies. In a broader sense, the term represents technical development and a state of mind. That is a way of thinking, an attitude, and a commitment to connected, global thinking to improve local, regional and global health using ICT.

According to the World Health Organization (WHO), Cyber health (also known as eHealth) consists of efficient and secure support that ICT provides to health and related areas. eHealth includes healthcare services, health surveillance, and documentation, as well as health education, knowledge, and research 7]. The following key components enable efficient and effective service to patients:

- Telemedicine: It is the provision of medical services at a distance. Telemedicine is applying advanced communications and computing technology to perform consultations, diagnoses, or surgeries remotely and in real-time. Moreover, as a service, it can benefit all patients in a healthcare system [8].

- Telehealth: Through videoconference, patients can be attended to evaluate specific ailments or diseases that the health worker can issue a diagnostic assessment or presumption and treatment. This technological resource enables the optimization of health care services, saving time and money. In this way, we facilitate access to patients from distant areas to have specialist care $([9])$.

- Electronic Clinical History is the information of each patient's health and clinical evolution in a digital format. With this information, medical professionals can take decisions or appropriate treatment, among others 10$]$.

\subsection{Triage}

Patient care in the Emergency area is done using a method called Triage [11,12]. This method classifies patients according to the patient condition to prioritize it over others of less severe condition [11]. Patient 
classification is identified by colors: red, orange, yellow, green, and blue, indicating priority in that order, respectively. Figure 1 presents a Triage scheme where each color has associated the patient's severity level and waiting time for their care. This information is communicated to patients and family members, minimizing service congestion.

Triage does not diagnose the patient's health status. However, it identifies the severity of the urgency of life-threatening patients. It determines the maximum waiting time to be treated for at the institution.

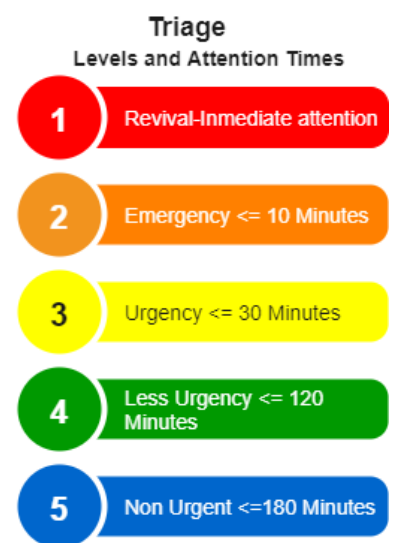

Figure 1: Triage levels with lead time

In this same context, the RAC (Reception, Assessment, and Classification) is complementary to the concept of Triage and incorporates the elements of the interpersonal relationship between the patient and the healthcare professional. RAC establishes an empathetic connection and focusing actions on patient service. RAC and Triage are used as synonyms in the RAC guide of the Ministry of Public Health of Paraguay. They are therefore adopted in this work $[13$. In DES, this classification of patients is used for the ordering according to the anamnesis performed, which means in clinical diagnosis: "Collection of relevant clinical information and the patient history". Anamnesis includes a personal and family history regarding the disease [14]. This work is carried out in DES, whose fundamental policy is to provide immediate medical care and seek stabilization of the patient's general condition.

Currently, there are different types of classification levels in the primary care system. They are standards applied according to its resources, infrastructure, and objectives to be covered to improve the quality of care and decrease the waiting time in the line. Health professionals recommend using at least five emergency levels within the Triage scale. The type of patient classified within each Triage level is similar at different scales [13. The meaning of the different Triage levels in terms of attendance and the maximum-desired time is outlined in Figure 1 .

\subsection{Electronic Health Records}

The Institute of Medicine (IOM) defines EHR as a longitudinal collection of electronic information about human health. Health information is defined as information relevant to an individual's health or healthcare information provided to an individual through any member of the health team [15.

These IOM reports also delineated eight-core functionalities of the EHR, and the Core Functionalities defined by the National Academy of Medicine (Formerly Known as the Institute of Medicine) are a) Health information and data; b) Result-based management; c) Order input/management; d) Decision support; e) Electronic communication and connectivity; f) Patient support; g) Administrative processes and reporting; h) Reporting and population health management 15 .

Therefore, some of this work helps identify possible usability problems in the patient's EHR, with their respective incidents that affect users during interaction with the SIGHU application. Therefore, SIGHU's behavior is analyzed through performance and efficiency tests detailed in this article.

\section{$2.3 \quad$ Research Related}

The Facultad de Ciencias Médicas of the UNA (FCM-UNA) manages the Hospital de Clínicas that is a Hospital School and conducts an ambitious automatization project of all hospital management. This system is called the Comprehensive Hospital Management System (SIGH in Spanish) [16]. The main objective of SIGH is the design, development, and implementation of the Healthcare, Administrative and Academic Management System whose modules are a) Patient Identification, which is responsible for centralized registration and identification of all patients, b) Stock and Pharmacy, controls and monitors the existence of 
medical supplies, c) Social Work, allows monitoring of the socio-economic situation of patients, (d) Assistance, facilitates the management of shifts and outpatient consultations, (e) Hospitalizations, manages the admissions and hospitalization of patients, f) Security, allows the management of user accounts and password management, (g) Emergency, management of medical emergencies, and (h) Laboratory and Imaging, SIGH interoperability with external software specialized in laboratory and imaging management.

The SIGH has not addressed the automatization of DES management. Therefore, the need for an application that complies with international and national standards has been determined for health personnel working in the Emergency area. Because the environment is a school hospital, the system should consider both the medical healthcare perspective and an academic viewpoint.

At the Hospital Foundation of the Community of Madrid (FHM), software was implemented that uses the same methodologies used for SIGHU and SIGH in the survey and development of a comprehensive system. This system is a complete set with several subsystems that use the standard procedures of each department where the system will be deployed. These subsystems communicate with each other using web interfaces to share information $[17$.

The Emergency Department Information System software from veEDIS is a medical management application used for EHR storage. As SIGHU provides health information to reduce errors, mitigate risk and improve overall patient outcomes in the emergency room [18. Unlike SIGHU, the veEDIS system only manages the storage of patient information. It does not manage the waiting list and intensive care within the Hospital Emergency Service.

Deaconess Hospital has implemented specific software for the Department of Emergencies of the Horizon Emergency Care. Created by emergency physicians, the system has management and follow-up capabilities for hospitalized patients. It also offers integrated nursing and medical documentation, automatic coding, clinical guidelines, order loading, and integrated prescriptions. It includes a tracking module that provides centralized and integrated means to monitor patients. This module allows nurses and other caregivers to access the most up-to-date patient information in seconds by providing real-time patient data. In turn, the physician can make prescriptions and control patients from a smartphone, laptop, tablet, or system with a browser 19]. It differs from SIGHU in that Horizon Emergency Care does not perform Triage monitoring of patients.

After reviewing the literature of emergency services medical systems, it is recognized that they help reduce the time of activities in the care process and paper use. The use of comprehensive systems ensures efficiency and efficiency. However, projects addressed with problems like the DES do not have the functionality to automate the standardized procedure known as RAC. Consequently, the need for an application such as the SIGHU that provides all these features to efficiently and effectively streamline the DES service is fully justified.

\section{Problem Statement}

The management of the DES is complex. It requires in clinical practice a demanding control of processes to ensure patient safety. The main problems encountered within the DES, which allow proposing a more effective solution to them, are as follows:

- Currently, approximately DES serves about 240 patients per day, with paper sheets-based management.

- DES physicians apply medical protocols manually using paper sheets. It represents many documents exposed to natural damage, such as fires, flooding, moisture, among others, or suffer loss in some times.

- This precariousness allows multiple users to have access to modifying the protocols, making it difficult to conduct an administration audit, that is, to know what was done, by whom, when, and why.

- DES's current working methodology leads to the loss of helpful information. It makes it difficult to obtain statistical data for future research, protocol evaluations, and physician decision-making. The DES needs statistical reports of mortality control, the number of patients moving to the various services that the Hospital has, and the average cost for each emergency patient. Obtaining this information timely will allow them to exercise better control over the entire process and mainly optimize the service for the benefit of their patients.

- The Emergency Department uses spreadsheets with numerous sheets that are difficult to handle and complete.

- Finally, an Emergency Services application on the domestic market is unknown. If it exists, it is not available academically for free. 


\subsection{Solution Proposal}

Considering the limitations raised within DES, the proposal for this work is to conduct the analysis, design, and implementation of a medical application called "SIGHU." It will facilitate the management and implementation of the procedure and treatment protocols, responding to the main problems of the DES. The application will allow the management of the data, reduce the use of resources and delimit costs during decision making. Furthermore, other departments can use it that applies protocols in its management, such as Pediatric Emergency. The main advantages that we seek to achieve with this automation are the following:

1. Manage the Electronic Patient Health Registry.

2. Use the Triage or RAC standard to facilitate service planning and management automatically.

3. Alert on the indications to be given to the patient to facilitate the physician's compliance with the protocol indications.

4. Prepare statistical reports for evaluation and decision-making.

5. Audit the administration of the emergency protocol in the DES.

6. Optimize the use of available resources for patient care.

7. Guide human resources management, improve workflow, functions and care circuits.

\subsection{RAC Attention Flow}

The flow within the primary care process (RAC and Triage) contains activities to be automated through a computer system. Since they are well defined and are manual processes for extracting clinical data before medical consultation, as seen in Figure 2, In addition, it is possible to facilitate reading of the information already processed when the system assigns to patient emergency level. According to the symptoms and measurements made to the patient, define this level is the most important to RAC. Patients within the systematized flow will be treated according to the standard, avoiding preferential care without Triage. If one software manages this process, no activity jump can be performed due to the system's restrictions. This change generates a robust attention mechanism where each activity has an actor. Each actor is responsible for the actions required to continue the flow [13].

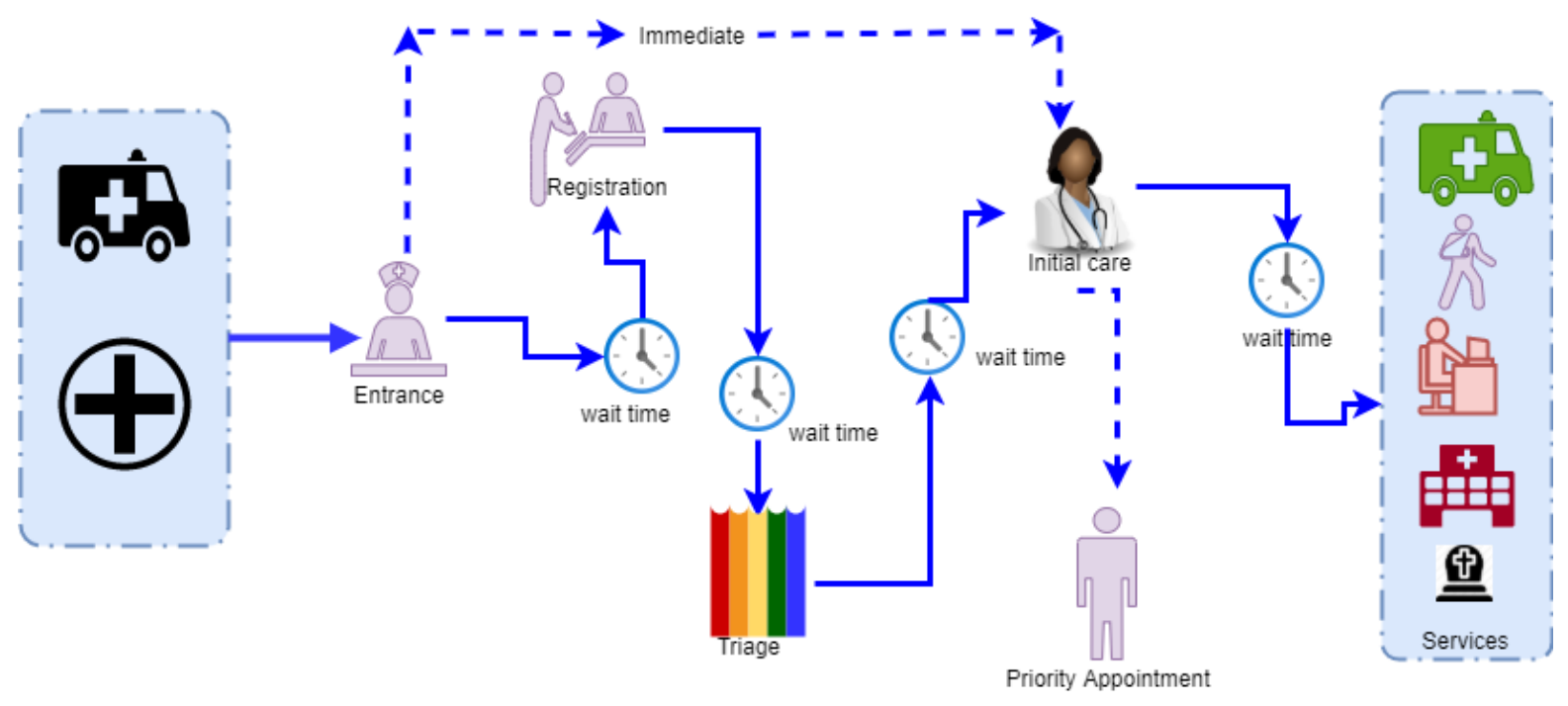

Figure 2: Emergency Steps

\subsection{SIGHU Design}

SIGHU lets doctors generate the protocols they use to treat the patient and enter their data during consultation or hospitalization in an intuitive and agile way. These protocols allow the data to be stored automatically. Statistical reports can be issued and generate helpful knowledge through some Artificial Intelligence algorithms. 
The development of SIGHU has been carried out using the Model-Driven Software Development (MDD) paradigm. Models are generated from the most abstract to the most concrete through transformation steps and refinements to reach the code by applying the last transformation 20. SIGHU has taken full advantage of this paradigm, such as improving developer productivity and reducing the use of available human and computational resources.

\subsubsection{SIGHU Components}

To fulfill these functions, SIGHU has the following components shown in Figure 3 . In the "RAC Sheets" item, RAC sheets are generated and managed. The nurse or receptionist begins the receiving-and-holding process of the patient by completing the data in the fields based on medical computer science standards. In the "SIGH Patients" item, the module communicates with the SIGH and manages patient and people data. In "Typifiers", it is possible to the loading measurable data by doctors or nurses. The "Infrastructure" allows the management of rooms and care units. To make the SIGHU medical and nursing sheet cutting configurations, it is entered the "Settings" item. Finally, in "Statistics," it is possible to obtain the statistical reports requested by the DES.

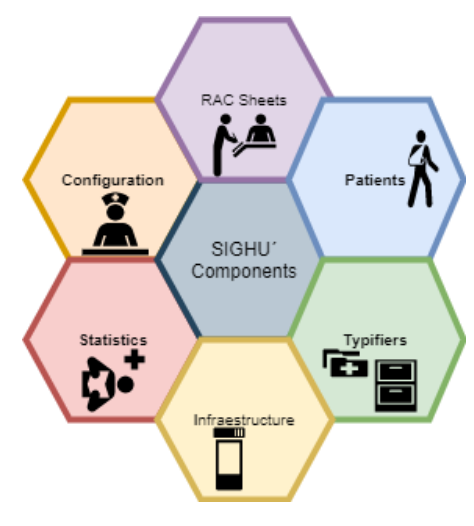

Figure 3: SIGHU Components

\subsection{SIGHU Integration with SIGH}

The SIGHU system is integrated into SIGH to facilitate comprehensive patient care for medical doctors. Figure 4 shows the structure of communication between systems, which specifies the modules involved in this integration and the database used.

The SIGHU module access is into the main menu of the SIGH Assistance module, which is enabled only for users who can access the SIGHU system. When we enter, we can see the different options to manage it. Like menu access, the role and charge permissions handle these options.

\subsection{User Activities in the SIGHU}

This section explains some functionalities performed by "key" users in the DES Emergency Management process.

Figure 5 shows the user "Head of Emergency or Head of Chair." The main functionality of this actor is to perform the management, configuration and maintenance of the Indication Sheets, Levels, Classification, Emergency Diagnostics, RAC Sheet Cutting, Recipes, Rooms, Images, inter-consultation Sheet and Evolution Sheet that are used in its Department. This user is the one who has access to all the SIGHU application options.

Figure 6 shows the tasks performed by "Doctor" or Resident Medical or a DES member. Alternatively, Figure 7 shows the functions performed by the user "Nurse": Manage RAC Sheet, Valuation History, Emergency Measurement, Nursing Sheet, Images.

In addition to these main users, the user "Receptionist" takes care of "Enter the RAC Sheet" and manages the patient data. The other user with essential functions is the "Informatics or Technology Manager, " responsible for managing user permissions and general data handled in the SIGH application.

\subsection{Software Development Environment}

The application development process uses the following technological tools, which have been chosen based on the MDD model. In Figure 8, we can see the tools used. 


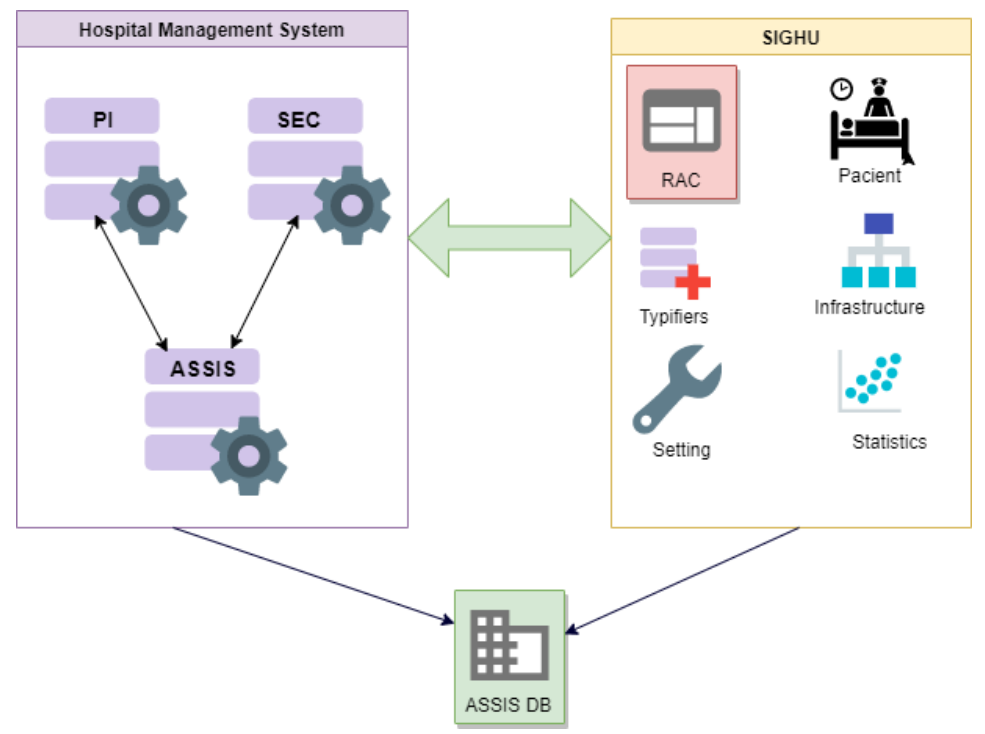

Figure 4: SIGHU integration with SIGH

\subsubsection{Framework Grails}

A framework is a workspace that helps us improve the productivity of software development to achieve the objectives sought during the analysis and research of the project. Provides programs, libraries, and other tools depending on the selected framework [16,21. Qualitative parameters that score each question within a survey 22 were measured for the choice of the framework. With the Grails framework, we achieve 149 points score, giving an acceptance percentage of $93 \%$, the acceptable minimum is $70 \%$. Another analyzed framework has a lower-than-average score, Django: with 72 points.

Grails is a free web application framework and developed in the Groovy language. It is a workspace that pursues the Don't Repeat Yourself (DRY) and Convention over Configuration (CoC) paradigm [23]. Fullstack developers opt for this type of productive work environment in a way that reduces failures, risks, and wasted time. To evaluate the Groovy programming language, we verified the characteristics of the language on the criteria established by Sebesta $[24$.

\subsubsection{Front-End Architecture}

The front-end architecture used free license tools. The integration was based on HTML 5 [25], CSS 3 26], Groovy Server Pages (GSP) [27], and JavaScript Technologies 28]. On the other hand, jQuery was used for calls to procedures Ajax within the client-to-server application. In addition, Bootstrap [29 has been used to design responsive views and Data Tables for deploying PivotTables within lists.

\subsubsection{Back-end Architecture}

The development of the Back End architecture was also based on free license tools. The development of drivers and services uses Groovy, while Java provides the function library. Gradle [30] has been used for plugin installation and configuration. The design of reports is based on Jasper Reports [31]. These reports are printed or downloaded on the client machine.

Finally, PostgreSQL [32] and [33 are used for database integration and architecture to map and relate to models and GORM [34]. The application server uses Tomcat [35] in version 8 and Java 8 for deployment within the system.

\section{Usability and Quality Analysis}

It is necessary to evaluate the software system as the development process progresses. In this way, developers try detecting detection as soon as possible. They have less impact on development time and effort. Analyzing the correct decision in an application is as or more important in the health area than in any other given that it works with human beings.

The factors to consider in this application are the functionality and technical aspects; by the scope of this work, they do not consider aspects of maintenance, support, and software costs. 


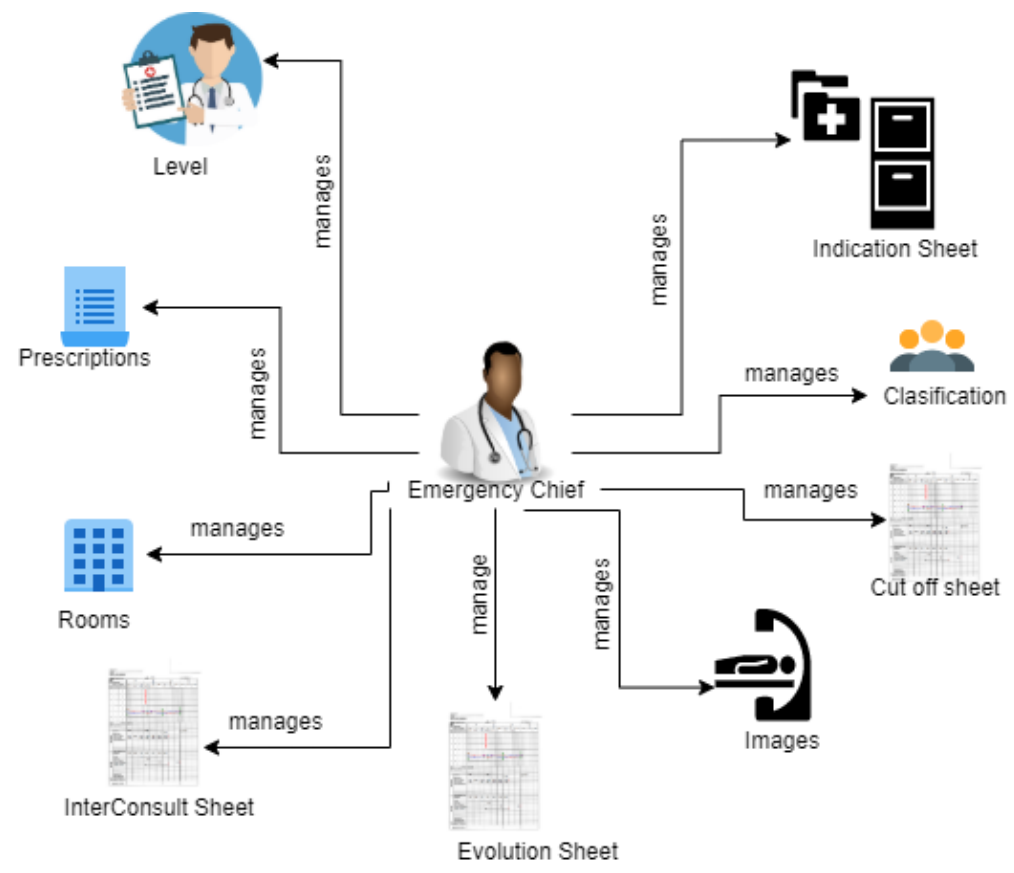

Figure 5: Emergency Chief

To ensure the correct use and a high degree of system acceptance, the developed application shall be obvious, evident, clear, and easy to understand. It shall be "usable" [36. In this context, it is crucial to define the meaning of "Usability." In this sense, Jakob Nielsen defined it as "a quality attribute that evaluates the ease of use of user interfaces" 37. Therefore, to determine whether the system is usable, there are techniques for performing formative or summative tests that determine the level of "Usability." The ease of using an EHR system has two components: intrinsic complexity and extrinsic difficulty. The intrinsic complexity reflects the complexity of the working domain and indicates the usefulness of the system. Extrinsic difficulty reflects the user's difficulty in using a user interface to perform a specific task and indicates system capability 38 .

According to the National Institute of Standards and Technology (NIST), there are different types of EHR. Therefore, the design of the user interface varies widely. The SIGHU system complies with the Clinical Approach and Software Platform types, as rated by NIST 39]. This software is developed to be explicitly used in the DES and is web-based. That is, it is accessible from any device that has a browser. According to system requirements, the Standard NIST Guide defines 343 applicable principles for the correct usability of software, mitigating the misuse of SIGHU functions and providing effective and safe use of the entire system.

\subsection{Usability Evaluation Using NIST Principles}

The user-centered design approach ensured the system's usability satisfactorily for its stakeholders, health personnel will be able to streamline their tasks thanks to the design that they themselves proposed during the development of the SIGHU. User-centric design cycles introduce some changes that strengthened the Usability of SIGHU and met good EHR design practices.

All categories of principles were included for verification of compliance with SIGHU system. The 335 design principles proposed by NIST were analyzed, but only a few of each selected category for evaluating the system.

NIST defines the steps for user-centered design: (a) User observations; (b) Risk identification; (c) Conceptual design; (d) Cognitive walkthrough; (e) User interview; (f) Mitigation development; (g) Detailed design; (h) Formative usability testing; (i) Verification; (j) Final design; (k) Summative usability testing.

The evaluation of NIST principles considered tables dividing them into two groups: applicable and not applicable in SIGHU. Each NIST category focuses on developing functions or promoting security resources. Some principles were not included because they refer to other design principles related to other systems.

Some design principles were not analyzed or applied because they are not part of the SIGHU system requirements list. They are unrelated to the type of application, or they are repetitive, or they are beyond the scope of this work. In the latter case, as future work, we propose functionalities. In summary, of the 335 design principles, 276 were applied, and 59 were not applied. The cycles of user-centered design can introduce some changes, which strengthened the usability of the SIGHU and was met with good design 


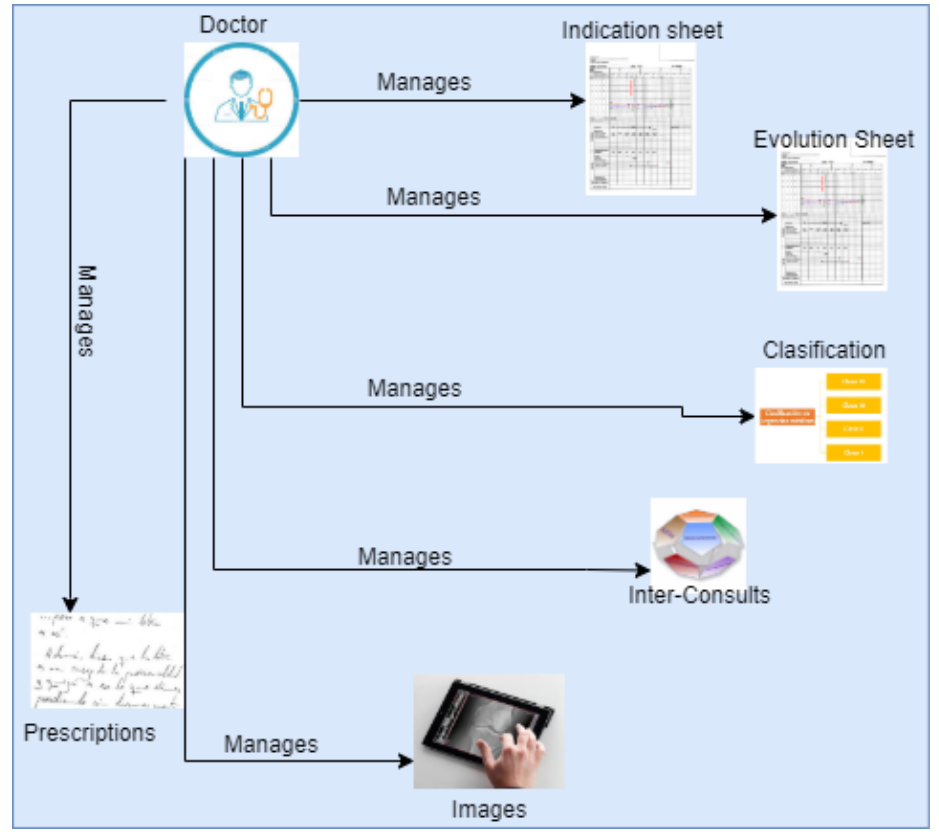

Figure 6: Doctor's tasks

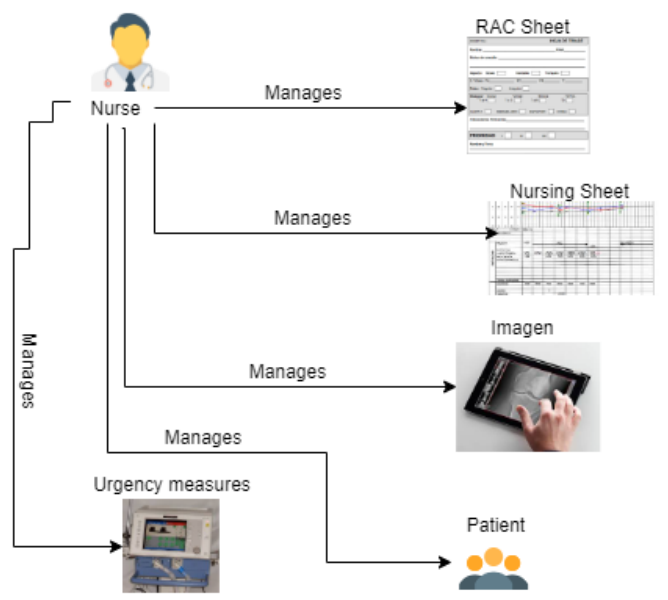

Figure 7: Nurse's Tasks

practices EHRs. In Figure 9 can see the percentage of principles that were applied and principles that were not applied.

\subsection{Heuristic Evaluation}

Performing the heuristic assessment involves having a small group of evaluators who examine the interface and judge its compliance with recognized usability principles. From the recognized principles, those that are part of the heuristic tests indicated by Nielsen have been taken. It also recommends that the number of effective evaluators for identifying the disadvantages is 3 to 5 evaluators [40]. The evaluators in this work are seven (7) professionals in the area of medical computer science. They worked on similar projects, so they know very well the workflow in the urgency.

Nielsen recommends the principles that were considered for this work are the following: (a) Visibility of the system' status; (b) Analogy between the system and the real world; (c) User control and freedom; (d) Consistency and standards; (e) Errors Prevention; (f) Acknowledgment instead of remembrance; (g) Flexibility and efficiency of use; (h) Aesthetic and minimalist design; (i) Helps users recognize, diagnose and recover from errors; (j) Help and documentation. Figure 10 shows that the benefits are greater than the costs for the heuristic evaluation with the optimal number of evaluators. By adding more evaluators reduces the benefit. The optimal number of evaluators in this example of the graph is 4, with benefits 62 times greater than costs. 


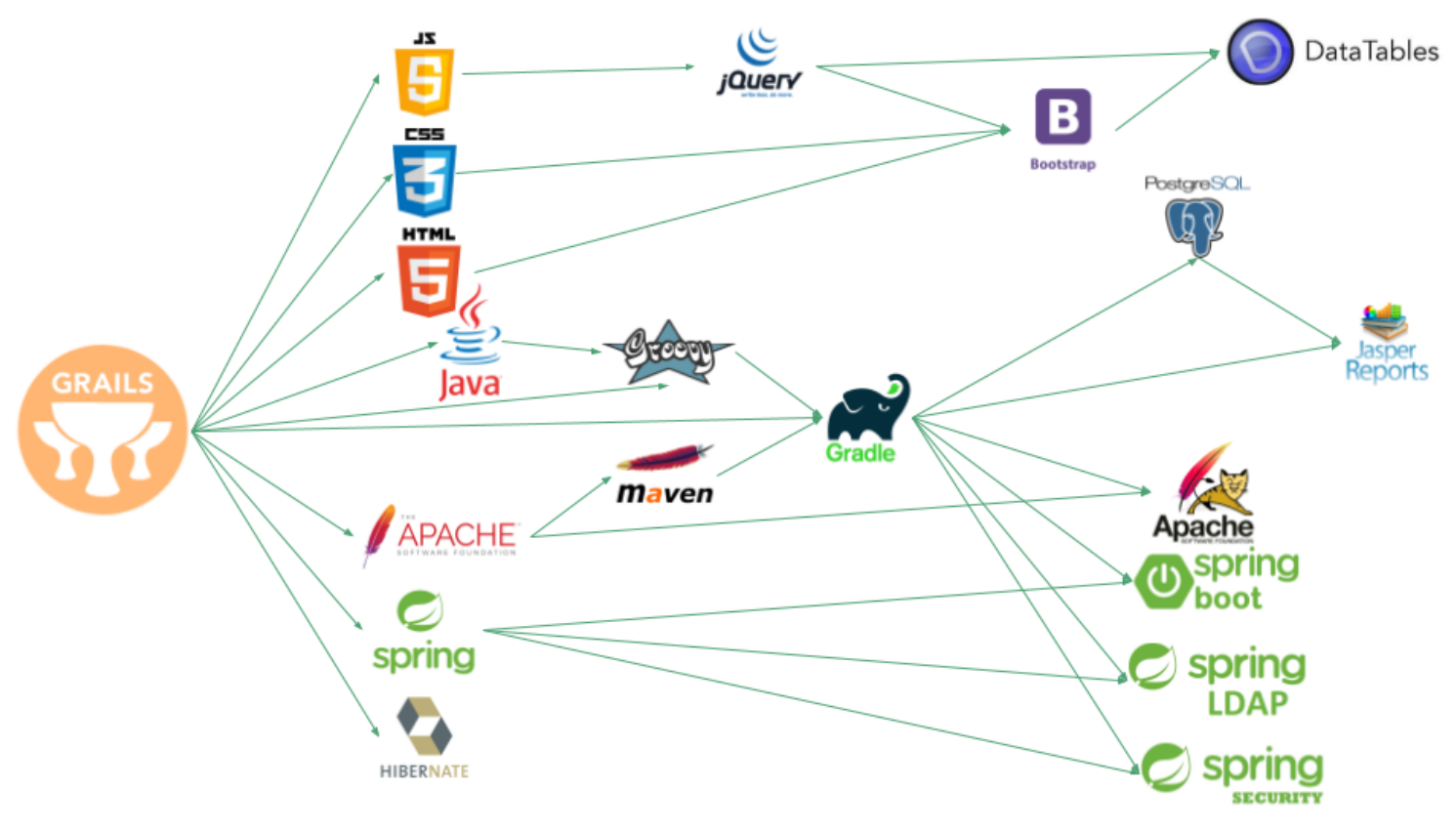

Figure 8: Tools used for SIGHU development

\section{Applied Design Principles}

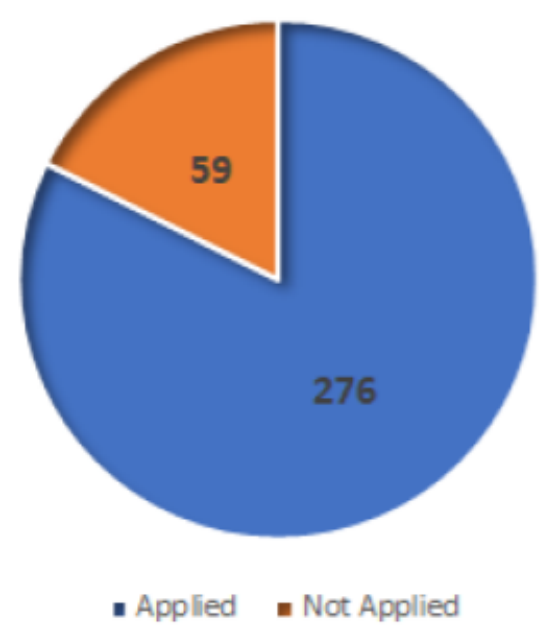

Figure 9: Applied Design Principles 
Table 1: Summary of usability flaws

\begin{tabular}{|c|c|c|c|c|}
\hline N. f/c & Description & Critical & Uncritical & Resolved \\
\hline 1 & Null Ocurrence Reports & $\mathrm{X}$ & & Yes \\
\hline 2 & Put progress graph on RAC sheet & & $\mathrm{X}$ & Not \\
\hline 3 & Indicate the fields required during loading & & $\mathrm{X}$ & Yes \\
\hline 4 & Jumping activities within the RAC & & $\mathrm{X}$ & Not \\
\hline 5 & Including hotkeys & & $\mathrm{X}$ & Not \\
\hline 6 & Improve labels translation & & $\mathrm{X}$ & Yes \\
\hline
\end{tabular}

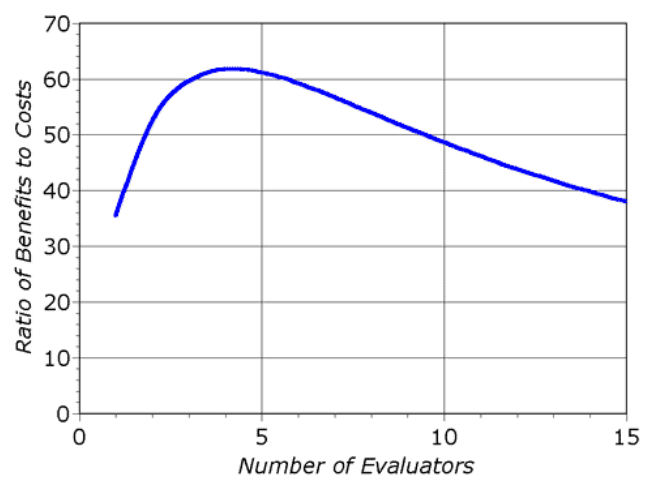

Figure 10: Benefits versus costs for heuristic evaluation 40

\subsubsection{Heuristic Exerciser for SIGHU}

The 7 evaluators perform an exercise with common tasks performed in the daily management within the DES, avoiding as much as possible the loss of time of the evaluator. Most of the evaluators did not belong to the group that faced the project. They performed tasks as dictated by the exercise or, in some cases, used the system guide to explore details of functionalities within the system.

After completing the exercise, the evaluators proceeded to fill out a form, where they comment on the shortcomings or lack of characteristics. In each item of the form, the evaluator can observe graphical examples. In addition, the evaluator's interpretation requires that it be immediate, without the need to waste time delving into more details of each characteristic. A short definition of each characteristic meaning is specified in each item. It gives the evaluator the ability to expand his field of view on the tasks he performed within the SIGHU system.

Heuristic assessment tests have identified some usability issues within automated DES management and design issues. Fixing these issues helps improve system usability to facilitate user accessibility. Table 1 summarizes these results. The test found six shortcomings or deficiencies (f/c) in the SIGHU system, which then in the new versions of the application have corrected the corresponding ones.

After the summary, we solve various deficiencies to improve the service provided by the SIGHU system. Only deficiencies 2, 4, 5 can not be solved because we cannot cut the workflow or skip activities. All activities are required and posed by easy access links from the RAC menu or sheet. Here, the functionality takes precedence over design because we cannot pause critical functionalities within DES.

For the SIGHU project, we perform the tests holistically based on the characteristics defined by Nielsen. In the Paraguayan market, the average salary for a web programmer is $\mathrm{U} \$ \mathrm{~S} 623$ and for a web designer is $\mathrm{U} \$ \mathrm{~S}$ 488. A quality assurance analyst is U\$S 600, the same as Nielsen's example. The difference that Nielsen adds is that it adds a fixed cost of $\mathrm{U} \$ S 4000$ for a quality assurance analyst who is in charge of evaluating the usability principles and the details of the requirement. To solve the usability failures found within a system requires two professionals, a designer, and a programmer. In total would cost U\$S 1111 monthly in a monthly payment (data based on the year 2020).

\subsection{Performance evaluation}

Among the evaluated performance measures, performance and unit tests (integrity and acceptance) are critical aspects. These measures are conducted before deploying the system in production. We consider conventional measures to evaluate the speed of the SIGHU given the WEB systems must not overload the infrastructure without overloading. 


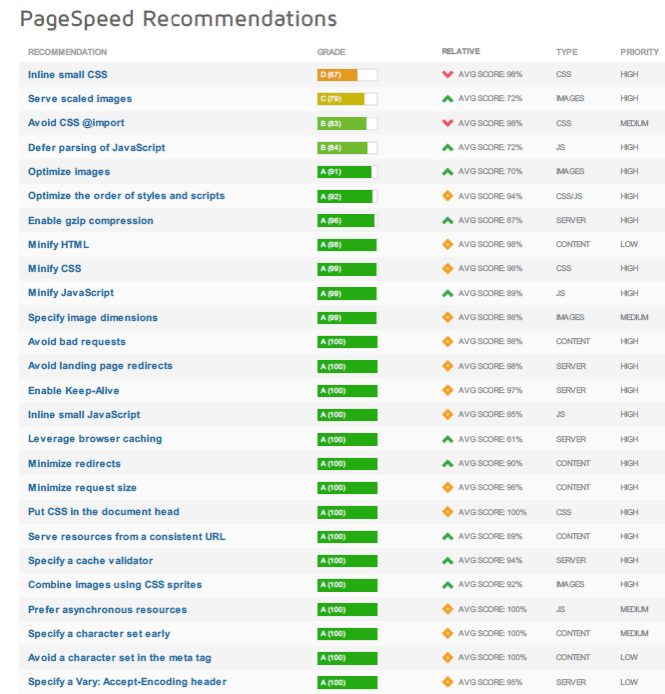

(a) PageSpeed detailed result

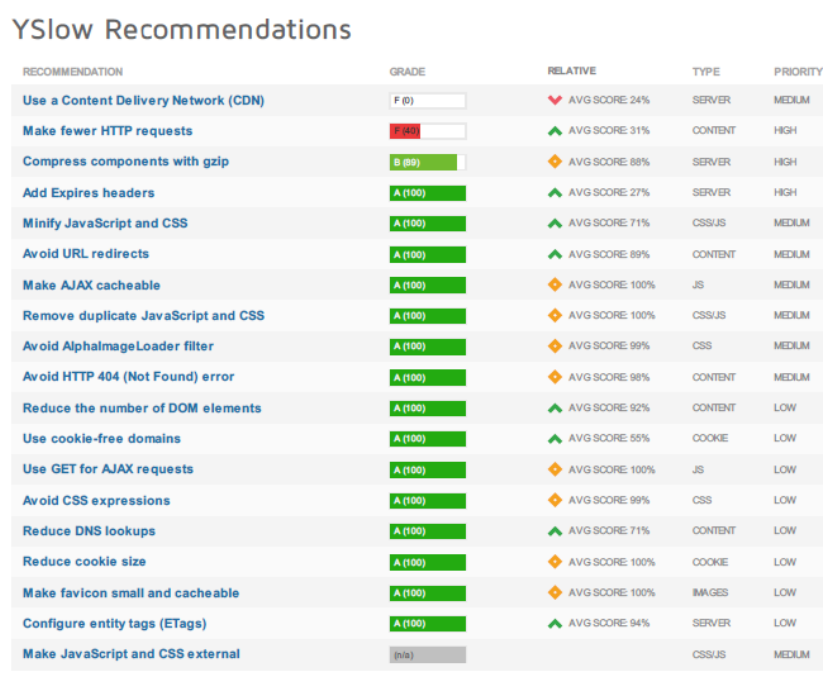

(b) YSlow detailed result

Figure 11: PageSpeed and YSlow Test

All the development of the SIGHU system was verified with the software testing processes. The development process has two well-defined objectives:

- To demonstrate to the developer and customer that the software meets their requirements.

- To discover defects in the software in which the software's behavior is incorrect, undesirable, or does not meet its specification.

Validation was performed using test strategy steps for web apps 41. Finally, the GTmetrix 42 performs tests on a fully integrated SIGHU. We consider evaluating two sets of rules within the system are the PageSpeed and YSlow rules.

Regarding the performance of unit tests, Grails framework has its tools for unit and integration tests. Within each test, we conducted the acceptance tests to verify the entire system and the tool's corroboration of the test report.

\section{Results and Discussion}

The requirement to develop computer applications that facilitate the realization of tasks to users has become a determining factor in software engineering. Therefore, for the DES, which serves a susceptible area of ehealth exist particular standards for development. In this section, we will briefly explain the experiment to evaluate the usability and quality of the software, following the rules of Nielsen and NIST.

According to the criteria applicable to the problem, we selected 276 of the 335 NIST design principles, corresponding to $80 \%$. Of the 276 principles considered, only $82 \%$ are applicable.

For the heuristic evaluation, we conclude that the SIGHU system is efficient, effective, and safe use in all functionality. The models have clear, analogous, and consistent visibility to the urgency workflow. Users have access to the guides simply, and the design of each workspace is aesthetically located for a minimalist environment.

For performance testing, PageSpeed's result measured in 2020 is an average of $88 \%$ in completed rules, indicating that the SIGHU system is above the current average, which was $72 \%$ measured in 2019 . This average may vary over time and measurements on the page. Figure 11a shows the characteristics analyzed by PagedSpeed.

YSlow analyzes web pages and suggests ways to improve performance considering a set of 34 optimization rules. The average result of which is $85 \%$ measured in 2020 [3]. Figure 11b shows the characteristics measured by YSlow. Figure 11 shows the good and average characteristics, and they outweigh the deficient ones. In this way, we indicate that the SIGHU system can operate, fulfilling the required parameters of performance or speed in the network of the Universidad Nacional de Asunción.

The unit tests use files with source code generated automatically with the Grails scaffold. Each file refers to each driver of the models used in the system; some sections of code were modified for testing because not everything is self-generated. The test found that some unit tests did not validate functionality held by 


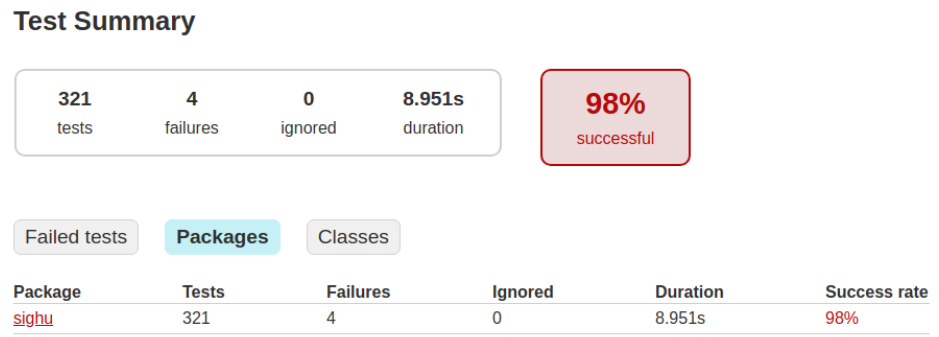

Figure 12: Unit Test Results

the RAC leaf controller. Those capabilities were verified by proof of acceptance, and the capabilities work correctly. Several parameters in sections of source code that the tool cannot validate yet. Therefore, it deploys errors within the validation. Figure 12 shows the percentage of acceptance of the source code by testing.

The integrity tests obtained results given in Figure 13. It is possible to observe the percentage of hits that SIGHU obtained for integrity tests.

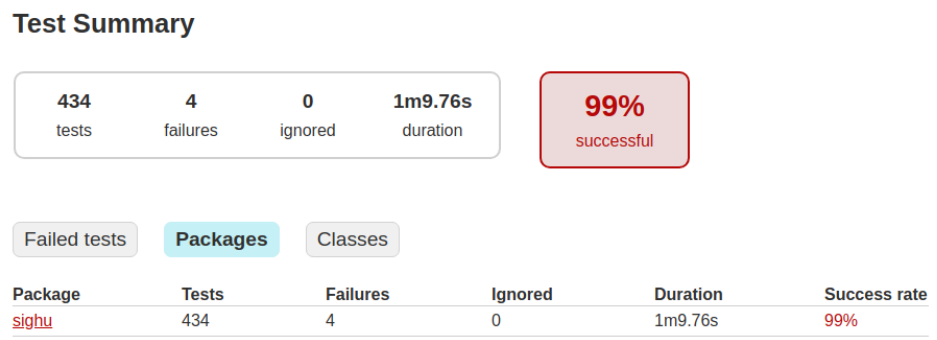

Figure 13: Integrity Test Results

According to these results, we can conclude that the system is optimal for use in the production environment. Great acceptance is achieved because in the evaluations conducted at SIGHU, good practices and principles are fulfilled.

\section{Conclusions and Future Work}

This work proposes the SIGHU system, whose objective allows the automation of managing the medical protocol indicated in the RAC Procedures standard, using good software engineering practices. It is the first system at the national level that focuses on Emergency Management installed within the computer network of the Hospital de Clínicas integrated with SIGH.

The information obtained using SIGHU allows doctors to more efficiently evaluate the outcome of treatment applied to patients concerning the manual management of DES doctors. The results of previous technical piloting show that SIGHU is robust and easy to maintain. No relevant technical problems were logged during this process. The acceptance of SIGHU has been positive on DES users, who stated that it is an easy-to-use, well-designed system that provides help proper interaction. These tests were performed throughout the software lifecycle.

The SIGHU system is constantly evolving and based on medical informatics standards. Its development remains in place, seeking to meet end-user requirements and incorporate the latest medical and informatics procedures.

As lines of future actions, we propose new features as follows:

- interoperability capabilities,

- automated input of large data sets,

- use of SIGHU in other medical units that manage urgency protocols such as the Pediatrics Unit,

- analysis of data collected by the software for medical research and decision making,

- capture, calculation, and comparison of parameters of measurements during the reception for automatic selection of the level of urgency, 
- automated calculation of maximum drug dosage, and

- use of barcodes within the system for early identification of patient RAC sheets and other functionalities.

\section{References}

[1] R. Salway, R. Valenzuela, J. Shoenberger, W. Mallon, and A. Viccellio, "Congestión en el servicio de urgencia: Respuestas basadas en evidencias a preguntas frecuentes," Revista Médica Clínica Las Condes, vol. 28, no. 2, pp. 220 - 227, 2017, tema central: Medicina de urgencia. [Online]. Available: http://www.sciencedirect.com/science/article/pii/S0716864017300366

[2] "Web site." [Online]. Available: https://www.med.una.py/index.php/hospital-hc/noticias-del-hospital/ 1392-hospital-de-clinicas-con-masiva-cantidad-de-pacientes-en-el-servicio-de-urgencias

[3] P.Alfieri, M. Benítez, N. Cardozo, S. Escobar, C. Cubells, O. Merlo, N. Meza, Z. Quintela, A. Paredes, S. Pavlicich, J. Quintilla, F. Rivas, M. Riveros, L. Martínez, A. Casanovas, and N. Tejada, Manual de Procedimientos. Recepción, Acogida y Clasificación (RAC), 2011.

[4] M. de Sanidad y Política Social Español, "Unidad de urgencias hospitalaria. estándares y recomendaciones," 2010. [Online]. Available: https://seup.org/pdf_public/pub/er_urgencias.pdf

[5] W. H. Organization et al., Informe sobre la salud en el mundo 2008: La atención primaria de salud, más necesaria que nunca. Ginebra: Organización Mundial de la Salud, 2008.

[6] G. Eysenbach, "What is e-health?" J Med Internet Res, vol. 3, no. 2, p. e20, Jun 2001. [Online]. Available: http://www.jmir.org/2001/2/e20/

[7] A. M. Informe de la Secretaría, "Cibersalud: informe de la secretaría," Organización Mundial de la Salud, Tech. Rep., 2005.

[8] F. Saigí-Rubió, J. Torrent-Sellens, C. Sáez, A. Kotzeva, and J. Villalobos, Marco de Implementación de un Servicio de Telemedicina. Organización Panamericana de la Salud, 052016.

[9] W. G. O. for eHealth, "Telemedicine: opportunities and developments in member states: report on the second global survey on ehealth," p. 93 p., 2010.

[10] MSPBS, "Quiindy integra la telemedicina a médicas," 04 2018. [Online]. Available: https://www.mspbs.gov.py/portal/15660/ quiindy-integra-la-telemedicina-a-sus-prestaciones-medicas.html

[11] R. Beveridge, "Canadian emergency department triage and acuity scale: implementation guidelines," CJEM, vol. 1, pp. S2-S28, 1999.

[12] N. B. Elkum, C. Barrett, and H. Al-Omran, "Canadian emergency departmenttriage and acuity scale: implementation in a tertiary care center in saudi arabia," BMC emergency medicine, vol. 11, no. 1, pp. $1-5,2011$.

[13] P.Alfieri, M. Benítez, N. Cardozo, S. Escobar, C. Cubells, O. Merlo, N. Meza, Z. Quintela, A. Paredes, S. Pavlicich, J. Quintilla, F. Rivas, M. Riveros, L. Martínez, A. Casanovas, and N. Tejada, Manual de Procedimientos Recepción, Acogida y Clasificación. RAC. Organización Panamericana de Salud, 2011, pp. $14-15$.

[14] Dorland, Diccionario Enciclopédico Ilustrado de Medicina. Interamericana-McGraw-Hill, 1992, p. 1489.

[15] I. of Medicine (US) Committee on Data Standards for Patient Safety, "Key capabilities of an electronic health record system," 2013. [Online]. Available: https://www.ncbi.nlm.nih.gov/books/NBK221802/

[16] G. Vázquez, M. Allegretti, M. E. García-Díaz, D. P. Pinto-Roa, A. Samudio, and y. D. Figueredo, "Aphope: Automation of pediatric hemato-oncological protocols of the hospital de clínicas," in 2019 XLV Latin American Computing Conference (CLEI), 2019, pp. 1-10.

[17] B. Escobar-Pérez, T. Escobar-Rodríguez, and P. Monge-Lozano, "Implantación de sistemas integrados para una gestión eficiente de los recursos en el ámbito hospitalario." Revista Cubana de Salud Pública, vol. 38, pp. 263-270, 2012. [Online]. Available: https://doi.org/10.1590/S0864-34662012000200009 
[18] P. Newswire, "DSS acquires veedis emergency department information system software," PR Newswire, 1 2019. [Online]. Available: https://www.dssinc.com/news/2019/1/9/ dss-acquires-veedis-emergency-department-information-system-software

[19] J. R. Staff, "Okc-based deaconess hospital adds emergency department-specific software," 62015. [Online]. Available: https://journalrecord.com/2005/06/22/deaconess-hospital-3/

[20] C. Pons, R. S. Giandini, and G. Pérez, Desarrollo de Software Dirigido por Modelos. Conceptos teóricos y su aplicación práctica. Editorial Universidad de La Plata, 2010, pp. 29-30.

[21] I. Sommerville, Ingeniería de Software. Pearson, 2011, pp. 431-434.

[22] M. A. Constanzo and S. I. Casas, "Usabilidad de framework web: identificación de problemas y propuesta de evaluación," in XXIV Congreso Argentino de Ciencias de la Computación (La Plata, 2018)., 2018.

[23] G. Rocher and J. Brown, The Definitive Guide to Grails. Apress, 2009.

[24] R. Sebesta, Concepts of Programmig Languages, 10th ed. Pearson, 2012, pp. 2-17.

[25] Mozilla Org, "Html5," https://developer.mozilla.org/es/docs/HTML/HTML5, 2020, accedido: 202005-02.

[26] Mozilla, "Css articles," https://hacks.mozilla.org/category/css/, 2020, accedido: 2020-05-02.

[27] Apache, "A multi-faceted language for the java platform," http://groovy-lang.org/, 2020, accedido: 2020-05-02.

[28] Pluralsight LLC, "Javascript," https://www.javascript.com/, 2020, accedido: 2020-08-02.

[29] GitHub, Inc., "Bootstrap," https://getbootstrap.com/, 2020, accedido: 2020-08-02.

[30] Gradle Inc., "Developer productivity engineering," https://gradle.org/, 2020, accedido: 2020-10-15.

[31] TIBCO Software Inc., "Jasper report," https://community.jaspersoft.com/, 2020, accedido: 2020-08-15.

[32] The PostgreSQL Global Development Group, "PostgreSQL: The World's Most Advanced Open Source Relational Database," https://www.postgresql.org/, 2020, accedido: 2020-10-15.

[33] Hibernate projects, "Hibernate ORM," https://hibernate.org/, 2020, accedido: 2020-10-17.

[34] Jinzhu, "The fantastic ORM library for Golang," https://gorm.io/, 2020, accedido: 2020-10-17.

[35] Apache Org., "Apache Tomcat," http://tomcat.apache.org/, 2020, accedido: 2020-10-20.

[36] R. Mack and J. Nielsen, Heuristic evaluation. Usability Inspection Methods. John Wiley and Sons, NY., 1994.

[37] J. Nielsen, "Usability 101: Introduction to usability (2012)," URL: http://www. nngroup. com/articles/usability-101-introduction-to-usability/[Accessed November 2016], vol. 9, p. 35, 2012.

[38] M. Walji and J. Zhang, "TURF: Toward a unified framework of EHR usability," Journal of Biomedical Informatics. Elsevier, vol. 44, pp. 1056-1067, 2011. [Online]. Available: https: //doi.org/10.1016/j.jbi.2011.08.005

[39] NIST, "Health it usability." [Online]. Available: https://www.nist.gov/programs-projects/ health-it-usability

[40] J. Nielsen, "How to conduct a heuristic evaluation," Nielsen Norman Group, vol. 1, pp. 1-8, 1995.

[41] R. Pressman, Ingeniería del Software. Un enfoque práctico. McGraw-Hill, 2010.

[42] GTmetrix, "General site speed faq," 2019, last access: February 2020. [Online]. Available: https://gtmetrix.com/faq.html\#faq-relative-score

[43] P. of marceldurand Hosted on GitHub Pages, "Optimización web con yslow," 2020. [Online]. Available: http://yslow.org/ 